\title{
Determinación de la actividad enzimática de la hexosaminidasa en fluidos biológicos
}

\author{
Marlén Rodríguez ${ }^{1}$, Angela Sánchez ${ }^{1}$, Mónica A. Gutiérrez ${ }^{2}$, Antonio J. Bermúdez ${ }^{2}$
}

\begin{abstract}
Resumen
Este estudio describe un método electroforético que emplea el sustrato fluorogénico 4metilumbelliferil 2-acetamida 2-deoxi-ß-D-glucopiranosido, para la determinación enzimática de la hexosaminidasa (HEX) en linfocitos de sangre periférica, líquido amniótico, suero y orina. Se propone que la electroforésis sirva como método confirmatorio del diagnóstico de la gangliosidosis GM2, ya que permite determinar la actividad enzimática de la HEX y hacer la diferenciación de las isoenzimas (HEX A y HEX B). La deficiencia de estas isoenzimas se detecta por disminución o ausencia de fluorescencia.
\end{abstract}

\section{Summary}

This study describes a method, that uses electrophoresis for the enzyme hexosaminidase (HEX), the substrate 4-metil-umbelliferyl 2-acetamide 2-deoxi-beta-Dglucopiranoside. We used samples from serum, urine and amniotic fluid, from normal people. We propose this method could be used as diagnostic for gangliosidoses GM2, because of the sensibility for the detection of isoenzymes HEX A and HEX $B$, and the capability to detect differences in fluorescence.

La hexosaminidasa (HEX) pertenece a la familia de las hidrolasas; esta enzima está presente en los lisosomas de todas las células nucleadas del organismo $(1,2)$.

La degradación lisosomal de la $\beta-\mathrm{N}$ acetilgalactosamina terminal del gangliósido GM2 requiere tres polipéptidos genéticamente distintos; las subunidades $\alpha$ y $\beta$ de la HEX $A$ y la proteína activadora GM2. Un defecto en alguno de los tres genes puede deteriorar el catabolismo del gangliósido (2-8).

Se presentan tres tipos de gangliosidosis GM2:

a. Enfermedad de Tay-Sachs o variante B; causada por una mutación en el cromosoma 15 , en el locus que codifica para la subunidad $\alpha$ de la HEX A. Los individuos con esta enfermedad poseen actividad de HEX A deficiente, pero, la HEX B es normal $(2,3,9)$.

b. En la enfermedad de Sandhoff o variante $O$, se presenta una mutación en el gen (cromosoma 5) que codifica para la subunidad $\beta$ Estos pacientes tiene deficien-te actividad de HEX $A$ y $B(2,3,9)$.

c. La variante $A B$ de la gangliosidosis $G M 2$ resulta de un defecto en el cromosoma 5 , en el gen que codifica para la proteína activadora. Estos pacientes tienen activa la HEX A y HEX B, pero, el catabolismo de GM2 es deficiente $(2,3,9)$.

Hasta el momento, se han encontrado más de cuarenta mutaciones alélicas diferentes en el locus de la subunidad $\alpha$ como responsables de

1 Universidad de los Andes, Santa Fe de Bogotá.

2 Laboratorio de Genética, Instituto Nacional de Salud, Santa Fe de Bogotá. 
enfermedades clínicamente diferentes. Todas están caracterizadas por disminución o ausencia de actividad de la HEX A (10-14).

Para la determinación de HEX, se han descrito técnicas electroforéticas que emplean el sustrato sintético 4-metilumbelliferil 2-acetamida 2 deoxi $\beta$-D-glucopiranósido. Al hidrolizarse este sustrato, se libera $\mathrm{N}$-acetilglucosamina, formándose 4-metilumbelliferona (fluorescente). Las bandas se visualizan a $366 \mathrm{~nm}(2,8,15)$.

En el presente estudio, se establecieron las condiciones óptimas para determinar la actividad enzimática de la HEX en diferentes fluidos biológicos de individuos normales.

\section{Materiales y métodos}

Se seleccionó una población de 15 individuos normales, de diferente edad, de ambos sexos y sin antecedentes personales ni familiares de patologías genéticas. Se tomaron $10 \mathrm{~mL}$ de sangre periférica heparinizada. Se recogieron 10 $\mathrm{mL}$ de la primera orina de la mañana, chorro medio. La obtención del líquido amniótico (aproximadamente, 20 a $40 \mathrm{~mL}$ ) fue mediante amniocentesis asistida por ecografía y enviada directamente al laboratorio.

\section{Obtención y procesamiento de leucocitos}

La capa de leucocitos se separó de la sangre total mediante centrifugación; a ésta se le adicionó agua destilada para hemolizar los hematíes. La isotonicidad se devolvió con solución salina $1,85 \%$. La liberación de las enzimas intralisosómicas se hizo por disrupción con sonicación. Posteriormente, se centrifugó y se utilizó el sobrenadante para la cuantificación de la enzima (16).

Los linfocitos se cultivaron según el protocolo descrito por Silva et al. (17). Después de 72 horas de incubación, se lavó el pellet con solución salina $0,9 \%$ y se continuó el mismo procedimiento que para los linfocitos de sangre periférica sin cultivar.

\section{Cultivo de fibroblastos}

Se efectuó según la técnica estandarizada para líquido amniótico en el Laboratorio de Genética del Instituto Nacional de Salud (18). Los fibroblastos se lavaron y resuspendieron en solución salina 0,85\%; la liberación de las enzimas se realizó por sonicación y se analizó el sobrenadante.

Se ensayaron diferentes tiempos de sonicación, los cuales variaron entre 2 y 30 segundos, con un número de pulsos que osciló entre dos y tres.

\section{Análisis electroforético}

La concentración del gel de agarosa inicialmente fue al 1\% (18), donde sólo se observó separación anódica; por tal motivo, se ensayaron geles de agarosa al 0,8 y $0,7 \%$ en solución amortiguadora de Tris $0,008 \mathrm{M} /$ ácido cítrico $0,004 \mathrm{M}, \mathrm{pH} 5,7$. Se sembraron $5,10,20$ y 40 microlitros $(\mu \mathrm{L})$ del sobrenadante obtenido después de la sonicación de linfocitos de sangre periférica, linfocitos cultivados y líquido amniótico; 5,10 y $25 \mu \mathrm{L}$ de suero y $10 \mu \mathrm{L}$ del control de migración constituido por albúmina bovina al $0,05 \%$ con azul de bromofenol como indicador. Se ensayaron tiempos de migración de 2,3 y 3,5 horas.

El revelado se hizo utilizando el substrato sintético 4-metilumbelliferil 2 acetamida-2 deoxi $\beta$-D-glucopiranósido en concentración de 0,54 miligramos en $1 \mu \mathrm{L}$ de solución amortiguadora de citrato $0,1 \mathrm{M}, \mathrm{pH} 4,5$. Se le adicionó la mezcla de substrato al gel y se dejó reaccionar a $37^{\circ} \mathrm{C}$ durante $30 \mathrm{~min}$. Para frenar e intensificar la reacción, el gel se colocó en una cámara de amoníaco por 2 horas. La identificación se realizó en cámara de luz ultravioleta para visualizar las isoenzimas como bandas brillantes a $366 \mathrm{~nm}$.

\section{Resultados}

Los resultados de la estandarización de la sonicación se observan en el cuadro 1.

\section{Análisis electroforético}

La cantidad de muestra se modificó de acuerdo con el fluido biológico que se estuviese analizando (cuadro 2). Los resultados de los ensayos que evaluaron las condiciones óptimas de voltaje e intensidad para la determinación de la enzima se muestran en el cuadro 3 . Al 
emplear el gel de agarosa al 1\%, no se vió buena migración catódica de la HEX; por tanto, se realizaron nuevos ensayos hasta obtener buena separación con diferenciación total de bandas, quedando la concentración óptima del gel en $0,7 \%$ (ver figura 1). Al comparar los diferentes tiempos de migración, se estableció como tiempo óptimo, 3 horas y $30 \mathrm{~min}$. En resumen, las condiciones adecuadas para la separación electroforética de la HEX se muestran en el cuadro 4.

Cuadro 1. Variaciones en los tiempos de sonicación.

\begin{tabular}{ccl}
\hline No. de pulsos & Ciclos & \multicolumn{1}{c}{ Resultados } \\
\hline 3 & $30 \mathrm{seg}$ & $\begin{array}{l}\text { No se observó fluorescencia } \\
\text { No se observó fluorescencia }\end{array}$ \\
3 & $20 \mathrm{seg}$ & $\begin{array}{l}\text { Fluorescencia de baja } \\
\text { intensidad }\end{array}$ \\
3 & $10 \mathrm{seg}$ & $\begin{array}{l}\text { Fluorescencia parcial } \\
\text { Completa fluorescencia y } \\
\text { buena direnciación de } \\
\text { bandas }\end{array}$ \\
\hline
\end{tabular}

Cuadro 2. Resultados de la cantidad de muestra necesaria para observar fluorescencia.

\begin{tabular}{|c|c|c|c|c|}
\hline & \multicolumn{4}{|c|}{ Cantidad de muestra en $\mu \mathrm{L}$} \\
\hline & 5 & 12 & 20 & 40 \\
\hline Líquido amniótico & - & - & + & ++ \\
\hline Fibroblastos & - & - & ++ & +++ \\
\hline Linfocitos & - & - & + & ++ \\
\hline Linfocitos cultivados & - & - & - & - \\
\hline Orina & - & - & - & - \\
\hline Suero & - & + & ++ & ++ \\
\hline
\end{tabular}

(-) No se oservó fluorescencia

(+) Fluorescencia de intensidad baja

$(++)$ Fluorescencia de intensidad intermedia

(+1+) Fluorescencia intensa

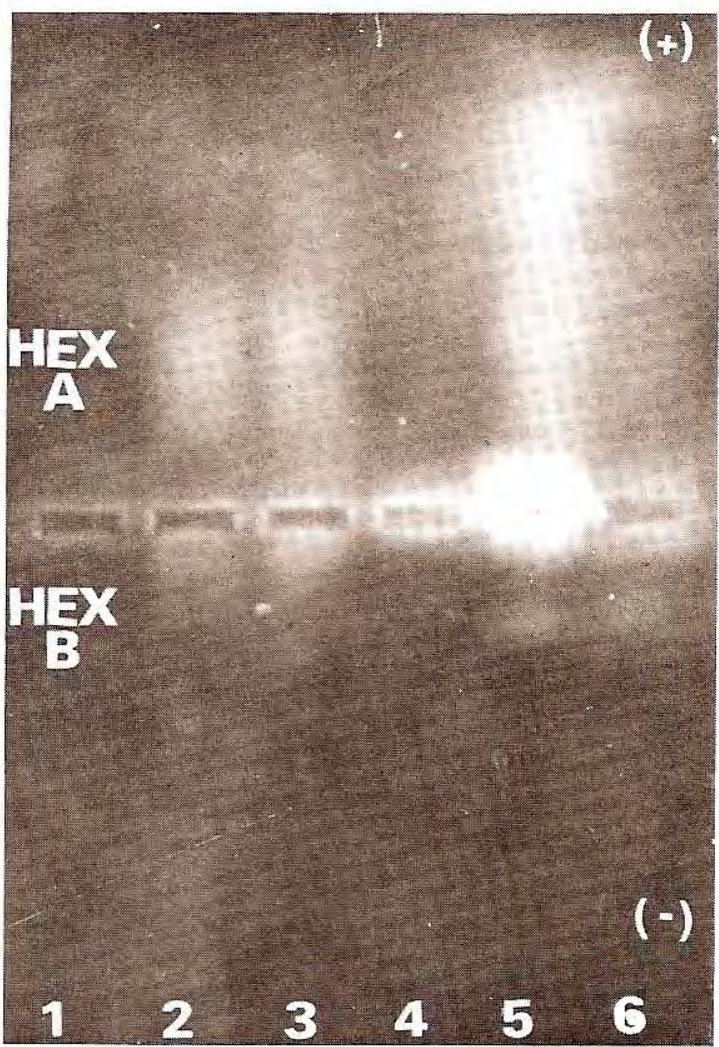

Figura 1. Determinación electroforética de la hexosaminidasa en muestras de pacientes normales.

Condiciones de la electroforesis: gel de agarosa al $0,7 \%$; solución amortiguadora de tris $0,008 \mathrm{M} /$ ácido cítrico 0,004 $\mathrm{M}$; $\mathrm{pH} 5,7$; voltaje: $200 \mathrm{v}$; intensidad: $8 \mathrm{~mA}$.

Carriles 1 y 4 : suero; carriles 2 y 3 : linfocitos de sangre periférica; carril 5 : fibroblastos de líquido amniótico; carril 6 : linfocitos cultivados.

No hubo interacción del sustrato con linfocitos cultivados, ni con el suero. Se vió fluorescencia producida por la interacción del sustrato con fibroblastos de líquido amniótico y linfocitos de sangre periférica. Obsérvese la diferenciación de las bandas anódicas (HEX A) y catódicas (HEX B) en los fibroblastos de líquido amniótico y linfocitos de sangre periférica. En el carril 6, se presentó un barrido inespecífico.

Cuadro 3. Condiciones de intensidad y voltaje para la determinación de hexosaminidasa.

\begin{tabular}{cccccccc}
\hline Vol. & Int. & \multicolumn{5}{c}{ Resultado } \\
\hline Voltios & m A & $\begin{array}{c}\text { Líquido } \\
\text { amniótico }\end{array}$ & Fibroblastos & Linfocitos & $\begin{array}{c}\text { Linfocitos } \\
\text { cultivados }\end{array}$ & Orina & Suero \\
\hline 25 & 6 & NS & NS & NS & NS & NS & NS \\
90 & 5 & BM, SSB & BM, SSB & BM, SSB & NS & NS & BM, SSB \\
200 & 8 & BM, DB & BM, DB & BM, DB & NS & NS & BM, DB \\
280 & 12 & MR & MR & MR & MR & MR & MR \\
\hline Vol: voltaje & Int: intensidad & & mA: miliamperios & & & \\
NS: no separación de bandas & & BM: buena migración & & & \\
SSB: sin separación de bandas & & DB: diferenciación de bandas & MR: migración rápida con mala resolución
\end{tabular}


Cuadro 4. Resumen de las condiciones óptimas para la separación electroforética de la HEX.

\begin{tabular}{|c|c|c|c|c|c|c|}
\hline & LA & FLB & $\mathbf{L}$ & LC & OR & SR \\
\hline Concentración del gel & $0,7 \%$ & $0,7 \%$ & $0,7 \%$ & - & - & $0,7 \%$ \\
\hline Sonicación & $2 p / 5 s$ & $2 p / 5 s$ & $2 p / 5 s$ & - & - & $2 p / 5 s$ \\
\hline Cantidad de muestra & $40 \mu \mathrm{l}$ & $20 \mu l$ & $40 \mu \mathrm{l}$ & - & - & $25 \mu l$ \\
\hline Voltaje (voltios) & 200 & 200 & 200 & - & - & 200 \\
\hline Intensidad (mA) & 8 & 8 & 8 & - & - & 8 \\
\hline Tiempo & $3 \mathrm{~h} 30 \mathrm{~m}$ & $3 \mathrm{~h} 30 \mathrm{~m}$ & $3 \mathrm{~h} 30 \mathrm{~m}$ & - & - & $3 \mathrm{~h} 30 \mathrm{~m}$ \\
\hline $\begin{array}{l}\text { LA: líquido amniótico } \\
\text { LC: linfocitos cultivados } \\
\text { p: pulsos }\end{array}$ & $\begin{array}{l}\text { LB: fibroblastos } \\
\text { OR: orina } \\
\mathrm{h} \text { : horas }\end{array}$ & & & & & \\
\hline
\end{tabular}

(-): no se pudo analizar electroforéticamente.

\section{Discusión}

Se utilizaron voltajes e intensidades bajas, como lo recomienda la literatura (19), obteniéndose migraciones lentas y difusas. Se realizaron pruebas aumentando el voltaje y la intensidad, pero, con mala resolución y sobrecalentamiento. Se decidió dejar como voltaje óptimo aquél que produjera buena definición de bandas y baja producción de calor. Según los irabajos realizados por Mosses et al. (20), se recomienda para la migración y separación de las isoenzimas un tiempo de 3 horas y $30 \mathrm{~min}$, en el cual se observó buena resolución de bandas tanto anódicas (HEX A) como catódicas (HEX B). El riesgo al incrementar el tiempo es que la enzima se degrade; sin embargo, esto no se observó aun con este largo tiempo de migración, en el cual juega papel decisivo el sistema de refrigeración.

Los resultados obtenidos con $40 \mu \mathrm{L}$ concuerdan con lo informado por Harris (19), cantidades menores de muestra fueron insuficientes para visualizar las dos bandas, excepto para fibroblastos en los cuales bastan sólo $20 \mu \mathrm{L}$ para producir buena fluorescencia. En todas las pruebas realizadas, se observó una mayor cantidad de la enzima en fibroblastos cultivados de líquido amniótico que en los otros fluidos biológicos; por esto, la cantidad de muestra necesaria fue menor.

\section{Conclusiones}

La electroforesis sirve para determinar la actividad enzimática de la hexosaminidasa y permite hacer la diferenciación de las isoenzimas (HEX A y HEX B). La deficiencia de las isoenzimas se puede detectar por la ausencia total o parcial de la fluorescencia, usando siempre controles normales. Esto se aplica como método confirmatorio de diagnóstico de la enfermedad de depósito lisosómico, gangliosidosis GM2.

Como los fibroblastos mostraron una buena definición de bandas y fluorescencia intensa, se puede utilizar la electroforesis en el diagnóstico prenatal de enfermedades con deficiencia de la hexosaminidasa.

El substrato sintético específico 4-metilumbelliferil 2-acetamida 2-deoxi $\beta$-D- glucopiranósido, empleado en este estudio, es una excelente opción para determinar cualitativamente la actividad enzimática de la hexosaminidasa, por su disponibilidad, pureza, sensibilidad y fácil manejo.

\section{Recomendaciones}

Como la actividad de la enzima es directamente proporcional a la intensidad de la fluorescencia de la bandas electroforéticas, se puede efectuar un análisis củantitativo a través de densitometría.

\section{Referencias}

1. Bieber RF. Pathologic findings in fetal GM1 gangliosidoses. Arch Neurol 1986;43:736-8.

2. Scriver C, Beaudet A, Sly W, Valle D, O‘Brien J. The metabolic basis of inherited disease. 6th ed. New York: McGraw-Hill; 1989:1807-38.

3. Shukry A, Goldman B, Shihab S, Peleg L. Prenatal and postnatal studies of a late infantile GM2 gangliosidosis in a family of Syrian origin: a possible B1 variant. Israel J Med Sci 1993;29(10):623-8. 
4. Sandhoif K, Andreae U, Jatzkewitz $H$. Deficient hexosaminidase activity in an exceptional case of TaySachs disease with additional storage of kidney globoside in visceral organs. Pathol Eur 1968;3:27885.

5. Sandhoff $K$, Harzer $K$, Wassle W, Yatzkewitz $H$. Enzyme alterations and lipid storage in three variants of Tay-Sachs disease. J Neurochem 1971;18:2469-89.

6. Conzelmann $E$, Sandhoff $K$. AB variant of infantile GM2 ganglioidosis: deficiency of factor necessary for stimulation of hexosaminidase A-catalyzed degradation of ganglioside GM2 and glicolipid GA2. Proc Natl Acad Sci USA 1978;75:3979-83.

7. Neufeld EF. Natural history and disorders of a lysosomal enzyme, beta-hexosaminidase. J Biol Chem 1989; 264:10927-30.

8. Mahuran DJ. The biochemistry of HEX A y HEX B gene mutations causing GM2 gangliosidoses. Biochem Biophys Acta 1991;1096:87-94.

9. Sewell A.C. Techniques in diagnostic human biochemical genetics. A laboratory manual. Wiley Liss, Inc.; 1991;219-31, 593-600.

10. Myerowitz R. Splice junction mutation in some Ashkenazi Jews with Tay-Sachs disease: evidence against a single defect within this ethnic group. Proc Natl Acad Sci USA 1988;85:3955-8.

11. Myerowitz R, Costigan C. The major defect in Ashkenazi jews with Tay-Sachs disease is an insertion in the gene for the alfa-chain of beta-hexosaminidase. J Biol Chem 1988;263:18587-9.

12. Paw BH, Kaback MM, Neufeld EF. Molecular basis of adult onset and chronic GM2-gangliosidoses in pa- tients of Ashkenazi jewish origin: substitution of serine for glycine at position 269 of the alfa-subunit of betahexosaminidase. Proc Natl Acad Sci USA 1989;86:2413-7.

13. Akli S, Chelly J, Mezard C, Gandy S, Kahn A, Poenaru L. A "G" to "A" mutation at position-1 of a $5^{\prime}$ splice site in a late infantile form of Tay-Sachs disease. J Biol Chem 1990;265:7324-30.

14. Navon R, Proia RL. Tay-Sachs disease in Moroccan jews: deletion of phenylalanine in the alfa-subunit of beta-hexosaminidase. Am J Hum Genet 1991;48:4129.

15. Devlin TM. Bioquímica. Tomo I. Barcelona: Editorial Reverte S.A.; 1985:500-8.

16. Sandoval A. Cuantificación e identificación de arilsulfatasa en orina y leucocitos (tesis). Bogotá: Universidad Javeriana; 1990.

17. Silva E, Crane C, Bermúdez AJ, Bueno ML, Pedraza X, Giraldo A. Citogenética humana. Manual de procedimientos. Bogotá: Instituto Nacional de Salud 1991.

18. Silva E. Estudio citogenético de vellosidades coriónicas y líquido amniótico en gestaciones de primero y segundo trimestre (tesis). Santa $\mathrm{Fe}$ de Bogotá: Universidad de los Andes; 1995:146-150.

19. Harris H. Handbook of enzyme electrophoresis in human genetics. Amsterdam: North Holanda; 1976.

20. Mosses SW, et al. Separation of N-acetyl B-D-hexosaminidase-isoenzymes from human brain and leucocytes by cellulose acetate paper electrophoresis. J Med Sci 1971;7(9):1086-9. 